\title{
Anemia e desnutrição maternas e sua relação com o peso ao nascer
}

\author{
Maternal anemia and undernowrishment and their relation to birth - weight
}

\author{
Odalis T.Sinisterra Rodriguez*, Sophia Cornbluth Szarfarc**, Maria Helena d'Aquino Benicio**
}

\begin{abstract}
SINISTERRA R., O.T. et al. Anemia e desnutrição maternas e sua relação com o peso ao nascer. Rev. Saúde públ., S. Paulo, 25: 193-7, 1991. Com o objetivo de avaliar a influência da anemia e desnutrição materna sobre o peso ao nascer, estudou-se uma amostra de 691 gestantes atendidas em maternidade exclusivamente assistencial do Município de São Paulo, SP (Brasil). Encontrou-se, no final da gestação, 29,2\% de gestantes anêmicas e 17,2\% de desnutridas, não tendo sido verificada associação entre essas duas deficiências nutricionais. $O$ papel favorável do serviço de assistência pré-natal no controle do estado nutricional da mulher não foi verificado, tendo sido observado que a anemia e desnutrição não estão associadas à assistência pré-natal. A incidência de baixo peso ao nascer (BPN) entre filhos de parturientes desnutridas foi $23,6 \%$, valor significativamente diferente $(p<0,005)$ do encontrado entre mulheres não desnutridas no final da gestação - $10,8 \%$. Comparando mulheres que iniciaram o processo gravídico desnutridas e que não se recuperaram com aquelas que se recuperaram, verifica-se risco relativo 2,8 vezes maior de conceptos com BPN entre as primeiras. Os resultados encontrados permitem ressaltar a necessidade de implementação nos programas de atendimento à gestante, de atividades relacionadas ao controle do estado nutricional da mulher.
\end{abstract}

Descritores: Anemia, epidemiologia. Estado nutricional. Peso ao nascer. Cuidados pré-natal.

\section{Introdução}

Entre os fatores que interferem na qualidade da gravidez e, conseqüentemente, no peso ao nascer, destacam-se as deficiências nutricionais.

A anemia é uma das deficiências nutricionais de maior importância durante a gestação, quer pela elevada prevalência com que ocorre, quer pelos efeitos adversos a ela associa$\operatorname{dos}^{2,7,11,14,16}$. Embora não conclusiva, a sua relação com o peso ao nascer tem sido verificada em diversos estudos. Harrison e Ibeziakos, em um estudo prospectivo, excluindo mulheres com complicações maternas e fetais e controlando a idade gestacional, observaram que os recém-nascidos de mães que tiveram anemia no transcurso e no final da gestação apresentaram médias inferiores de peso ao nascer. Mitchell e Lerner ${ }^{9}$, analisando dados retrospectivos de 1.080 gestantes americanas de classe média, encontraram forte relação entre níveis de hemoglobina materna e peso ao nascer.

A relação entre a desnutrição protéica-energética e o peso ao nascer vêm sendo avaliados desde meados do século ${ }^{1,19}$. Rosso ${ }^{13}$, a partir

\footnotetext{
* Ministério da Saúde, Panamá

** Departamento de Nutrição da Faculdade de Saúde Pública da Universidade de São Paulo - São Paulo, SP - Brasil Separatas/Reprints: S.C. Szarfarc - Av. Dr. Arnaldo, 715 - 01255 - São Paulo, SP - Brasil.
}

de uma revisão de estudos observacionais, destaca que na espécie humana a linha de abastecimento fetal é infuenciada por diferentes mecanismos que dependem da disponibilidade de nutrientes. Se a ingestão dietética for insuficiente e se os estoques de nutrientes da mãe estiverem baixos, o feto precisará recorrer às reservas préconcepcionais para se suprir, ocasionando comprometimento do binômio materno-fetal. De forma geral, a diminuição na ingestão de nutrientes pode provocar alterações nos mecanismos maternos de adaptação à gravidez e desacelerar o transporte de nutrientes.

Não obstante a anemia e desnutrição estarem bem caracterizadas como fatores isolados de risco de eventos indesejáveis na gestação e, como tal, estarem inseridas nos programas de atendimento à gestante, pouco se sabe sobre o peso ao nascer e menos ainda sobre a eficácia do pré-natal no controle dessas variáveis. O presente trabalho foi realizado com vistas a estudar essas relações.

\section{Metodologia}

A população de estudo foi composta por 691 gestantes assistidas na sala de parto do Hospital Maternidade "Leonor Mendes de Barros" (HMLMB), Município de São Paulo, cujo produto de gestação foi nascido vivo. Foram excluídos os partos gemelares. 
As variáveis estudadas foram: na gestante - concentração de hemoglobina, peso e altura no final da gestação, peso pré-gestacional, freqüência a serviços de pré-natal; no recém-nascido - peso ao nascer.

O diagnóstico da anemia foi feito pela medida da concentração de hemoglobina ( $\mathrm{Hb})$. $\mathrm{O}$ sangue venoso utilizado para a dosagem foi $o$ colhido na sala de pré-parto, em tubo contendo o anti-coagulante EDTA. O nível crítico utilizado foi o proposto pela Organização Mundial da Saúde-OMS ${ }^{10}$, que classifica como anêmicas, gestantes com concentração de hemoglobina inferior a $11,0 \mathrm{~g} / \mathrm{dl}$. O diagnóstico desnutrição utilizou padrões elaborados por Siqueira e col. ${ }^{17}$ que considera a relação peso/altura associada à idade gestacional.

Para análise dos dados foram utilizados o teste de associação quiquadrado $\left(x^{2}\right)$ e a medida do risco relativo (RR) que expressa a intensidade da associação ${ }^{6}$. $O$ nível de significância adotado foi alfa $=5 \%$ (quiquadrado crítico $=$ $3,84)$.

\section{Resultados e Comentários}

As mulheres que utilizavam os serviços obstétricos oferecidos pelo HMLMB, entidade de caráter exclusivamente assistencial, são, na maior proporção, aquelas sobre quem recaem condições ambientais adversas e pequena disponibilidade financeira e de recursos médico-assistenciais. Cerca da metade delas $(54 \%)$ são solteiras e, dentre o grupo, $81,3 \%$ não completou o primeiro grau escolar (antigo primário e ginásio).

A maior parte da população estudada $(88 \%)$ matriculou-se em serviços de assistência pré-natal (Tabela 1), embora metade delas não o tenha freqüentado com a concentração mínima recomendada de 5 consultas ${ }^{6}$. Os serviços de pré-natal procurados foram exclusivamente aqueles oferecidos pela Rede de Serviços de Saúde. Vale a pena ressaltar que este percentual de matrículas ao pré-natal é bem mais elevado que os $38 \%$ referidos na mesma instituição, em $1972^{20}$.

Tabela 1. Distribuição das gestantes segundo freqüência aos serviços de assistência pré-natal.

\begin{tabular}{ccc}
\hline N. de Consultas & No & $\%$ \\
\hline 0 & 84 & 12,0 \\
$1 \mathrm{a} 4$ & 281 & 42,1 \\
$5 \mathrm{e}+$ & 316 & 45,9 \\
\hline
\end{tabular}

Embora tenha sido implementado o atendimento pré-natal para a população de gestantes que constitui a clientela do HMLMB, a incidência de baixo peso ao nascer (BPN) e de peso inadequado ao nascer (PIN), mantiveram-se semelhantes às observadas em 1972 (Tabela 2).

Tabela 2. Distribuição de recém-nascidos segundo peso ao nascer em dois inquéritos realizados em $1972^{20}$ e do presente estudo.

\begin{tabular}{lccc}
\hline Inquierito & Amostra $\%$ BPN & $\begin{array}{c}\% \text { PIN } \\
<2.500 \mathrm{~g}\end{array}$ & $\begin{array}{c}2.500-3.000 \mathrm{~g} \\
\end{array}$ \\
\hline Szarfarc $^{20}(1972)$ & 248 & 12,4 & 25,1 \\
do Estudo & 691 & 12,9 & 28,8 \\
\hline
\end{tabular}

Mesmo assim, conforme o esperado, foi verificada associação estatisticamente significante $\left(x^{2}=5,29\right)$ entre assistência pré-natal e peso ao nascer. A incidência de baixo peso entre filhos de mulheres que não frequientaram o prénatal (menos de 5 consultas) foi aproximadamente o dobro da observada entre o grupo com prénatal $(R R=1,87)$.

O efeito positivo da assistência pré-natal, no entanto, não foi encontrado em relação à prevalência de anemia e de desnutrição, entre as parturientes (Tabela 3 ).

Tabela 3. Distribuiçāo das parturientes segundo deficiências nutricionais e assistência pré-natal.

A - Anemia

\begin{tabular}{cccc}
\hline Atenção & \multicolumn{2}{c}{$\mathrm{Hb}<11,0 \mathrm{~g} / \mathrm{dl}$} & \multirow{2}{*}{$\begin{array}{c}\text { Total } \\
\text { Pré-Natal }\end{array}$} \\
\cline { 2 - 3 } (N.: de Consultas) & $\mathrm{Sim}$ & Não & \\
\hline$<5$ & 112 & 256 & 368 \\
$>=5$ & 77 & 239 & 316 \\
\hline Total & 189 & 495 & 684 \\
\hline
\end{tabular}

$\times 2=n \cdot s$

B - Desnutrição

\begin{tabular}{|c|c|c|c|}
\hline \multirow{2}{*}{$\begin{array}{c}\text { Atenção } \\
\text { Pré-Natal } \\
\text { (N. de Consultas) }\end{array}$} & \multicolumn{2}{|c|}{ Desnutridas } & \multirow{2}{*}{ Total } \\
\hline & Sim & Não & \\
\hline $\begin{array}{c}<5 \\
>=5\end{array}$ & $\begin{array}{l}46 \\
46\end{array}$ & $\begin{array}{l}209 \\
235\end{array}$ & $\begin{array}{l}255 \\
281\end{array}$ \\
\hline Total & 92 & 536 & $536^{*}$ \\
\hline
\end{tabular}

O grande número de casos sem informação (155), foi conseqüência da falta, na rotina do serviço, da ativida. de de pesagem da parturiente.

A independência entre as variáveis apresentadas na Tabela 3 destaca a necessidade de incentivar, dentro do Programa de Atendimento à Gestante (PAG) ${ }^{15}$ atividades referentes ao controle e/ou prevenção de deficiências nutricionais.

A exemplo do referido em outros estudos epidemiológicos ${ }^{2,4,8,12}$ foi constatada a efetividade 
da suplementação em ferro no controle da anemia ferropriva (Tabela 4).

Tabela 4. Parturientes com pré-natal segundo ingestāo de suplemento de ferro e anemia.

\begin{tabular}{cccc}
\hline $\begin{array}{c}\text { Ingestảo de } \\
\text { Ferro* }\end{array}$ & \multicolumn{2}{c}{$(\mathrm{Hb}<11,0 \mathrm{~g} / \mathrm{dl})$} & \multirow{2}{*}{ Total } \\
\cline { 2 - 3 } & $\mathrm{Sim}$ & $\mathrm{Não}$ & \\
& $\mathrm{N}:(\%)$ & N. $(\%)$ & N.\% \% \\
\hline Não & $46(43,8)$ & $59(56,2)$ & $105(35 \%)$ \\
Sim & $41(21,0)$ & $154(70,0)$ & $195(65 \%)$ \\
Total & 87 & 213 & 300 \\
\hline
\end{tabular}

* 16 sem informação

$x 2=16,12$

De acordo com o PAG ${ }^{15}$, as gestantes devem ingerir, diariamente, a partir do 2. trimestre de gravidez, $60 \mathrm{mg}$ de ferro. Os serviços de pré-natal deveriam distribuir esse suplemento na forma de comprimidos de sulfato ferroso. Chama a atenção, no entanto, o fato de que $35 \%$ das mulheres que freqüentaram adequadamente o pré-natal não receberam o suplemento.

Assim, se por um lado fica patente que a freqüência ao serviço de pré-natal não é garantia do recebimento e ingestão do sal de ferro, por outro lado, fica justificada a não existência de associação entre anemia e freqüência a serviços de pré-natal.

Ressalta-se na Tabela 5 a ausência de associação entre anemia e desnutrição maternas. Este fato sugere que o consumo energético apropriado não assegura a adequação do consumo de nutrientes específicos, com destaque para o ferro. Embora ausente na população estudada, a relação anemia e desnutrição é inerente ao recém-nascido uma vez que a concentração de ferro dessa criança é proporcional ao seu peso $(75 \mathrm{mgFe} / \mathrm{kg} \text { de peso) })^{7}$.

Tabela 5. Distribuição das parturientes segundo anemia $(H b<11,0 \mathrm{~g} / \mathrm{dl})$ e desnutrição maternas.

\begin{tabular}{|c|c|c|c|}
\hline \multirow{2}{*}{ Desnutrição } & \multicolumn{2}{|c|}{ Anemia } & \multirow{2}{*}{ Total } \\
\hline & Sim & Não & \\
\hline $\begin{array}{l}\text { Sim } \\
\text { Não } \\
\text { Total }\end{array}$ & $\begin{array}{l}24 \\
124 \\
148\end{array}$ & $\begin{array}{l}69 \\
324 \\
393\end{array}$ & $\begin{array}{l}93 \\
448 \\
541\end{array}$ \\
\hline
\end{tabular}

Estudando o papel das deficiências nutricionais como fatores determinantes do baixo peso em recém-nascidos, verificou-se a não associação entre anemia e peso ao nascer. Essa associação não ocorreu mesmo diferenciando o recémnascido de baixo peso (prematuro e a termo) e/ou apenas os casos de anemia severa. No entanto, foi observada associação, estatisticamente significante, entre desnutrição no final do período gravídico e baixo peso ao nascer (Tabela 6).
Tabela 6. Distribuição das parturientes segundo desnutriçảo e incidência de baixo peso ao nascer.

\begin{tabular}{lccc}
\hline Peso do & \multicolumn{2}{c}{ Desnutridas* } & \multirow{2}{*}{ Total } \\
\cline { 2 - 3 } RN $(\mathrm{g})$ & Sim & Não & \\
\hline$<2.500$ & 22 & 38 & 60 \\
$>=2.500$ & 71 & 313 & 384 \\
Total & 93 & 351 & 444 \\
\hline
\end{tabular}

$x 2=9,29 p<0,005$

* Excluidas as obesas.

Diferentemente do que se observou para anemia, a proporção de crianças com peso ao nascer inferior a $2.500 \mathrm{~g}$ foi cerca de dez vezes superior entre prematuros quando comparados aos recém-nascidos a termo.

$O$ risco de BPN modifica-se em função do tipo de evolução do estado nutricional durante a gestação (Tabela 7).

Tabela 7. Evolução* do peso gravídico e incidência de baixo peso ao nascer $(<2.500 \mathrm{~g})$.

\begin{tabular}{|c|c|c|}
\hline \multicolumn{2}{|c|}{ Estado Nutricional ${ }^{17}$} & \multirow{2}{*}{$\begin{array}{c}\text { Incidéncia } \\
\text { de } \\
\text { BPN }(\%)\end{array}$} \\
\hline Situaçāo inicial & Evolução & \\
\hline Normal & $=$ & 11 \\
\hline Normal & + & 11 \\
\hline Normal & & 13 \\
\hline Normal & & 23 \\
\hline Desnutrida & $=$ & 25 \\
\hline Desnutrida & + & 25 \\
\hline Desnutrida & & 38 \\
\hline Desnutrida & ++ & 12 \\
\hline
\end{tabular}

"Evolução = variação da adequação do peso final e inicial (adeq. F-adeq. I) onde

adeq. $F=$ (peso no parto/peso mínimo adeq. no parto) $\times 100$ adeq. 1 = (peso pré-gravídico/peso mínimo normal para a mulher) $\times 100$.

Resultado da Evolução

Significado

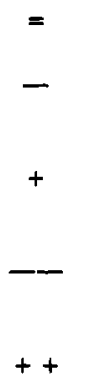

Não houve variação. A diferença foi inferior a $3 \%$. Variaçāo negativa e manutenção na classificaçāo do estado nutricional.

Variação positiva e manutenção na classificação do estado nutricional.

Variação negativa e mudança na classificação do estado nutricional.

$++\quad$ Variação positiva e mudança na classificação do estado nutricional.

A importância de atenção pré-natal no controle da desnutrição durante a gestação, fica evidenciada com os dados apresentados na Tabela 7.

Comparando, entre si, diferentes evoluções do estado nutricinal na gestação (Tabela 8 ), verifica-se que, se a desnutrição estiver corrigida no final do processo, o risco de baixo peso ao nascer diminui, tornando-se semelhante ao das mu- 
lheres que mantiveram normalidade de peso durante o decorrer de toda a gestação.

Tabela 8. Estimativa dos riscos relativos de baixo peso ao nascer $(<2.500 \mathrm{~g})$ obtidos comparando diferentes evoluçōes do estado nutricional materno.

\begin{tabular}{lcc}
\hline \multicolumn{2}{c}{ Evolução nutricional } & $\begin{array}{c}\text { Risco Relativo } \\
\text { de } \\
\text { BPN }\end{array}$ \\
\hline Situação A (n) & Situação B (n) & 2,60 \\
DI-DF (42) & NI-NF (284) & 2,98 \\
DI-DF (42) & DI-NF (33) & 2,20 \\
NI-DF (49) & NI-NF (284) & 1,18 \\
DI-DF (42) & NI-DF (49) & 0,90 \\
\hline DI-NF (33) & NI-NF (284) & \\
\hline
\end{tabular}

$\mathrm{DI}=$ Desnutrição inicial $\quad \mathrm{NI}=$ Normalidade inicial DF = Desnutrição final NF $=$ Normalidade final

$(n)=n$. de gestantes com determinada evolução nutricional.

A influência do peso materno no final da gravidez sobre o peso ao nascer também havia sido observada por Siqueira ${ }^{18}$ que assinalou ser o peso no final da gravidez um indicador importante do risco de peso inadequado. Também Barros e col. ${ }^{3}$, verificaram que o peso no final da gravidez é a variável que mais se relaciona com o peso do concepto.

\section{Conclusões}

O estudo da situação nutricional da gestante no que se refere à anemia e à desnutrição e sua relação com o peso ao nascer, em maternidade exclusivamente assistencial, permitiu as seguintes conclusões:

- A prevalência de anemia no final da gravidez foi de $29,2 \%$ e a de desnutrição, $17,2 \%$;

- Não foram observadas associações entre anemia e desnutrição e entre essas deficiências nutricionais e freqüência a serviços de atenção pré-natal. Verificou-se que a freqüência regular ao serviço de assistência pré-natal não é garantia de recebimento de suplemento de ferro. Entre as gestantes beneficiadas com esse suplemento, a prevalência de anemia foi estatisticamente menor do que entre as outras;

- Não foi encontrada associação entre anemia e baixo peso ao nascer;

- Foi observada associação estatisticamente significante entre desnutrição e baixo peso ao nascer, sendo que o risco relativo de baixo peso ao nascer entre filhos de parturientes desnutridas é, praticamente, o dobro do apresentado por filhos de não desnutridas. A recuperação da gestante desnutrida iguala o seu risco de conceptos de baixo peso ao de mulheres normais.

Destaca-se a necessidade de implementar nos serviços de assistência pré-natal atividades relacionadas ao controle da desnutrição e da anemia, com vistas a melhorar a qualidade do processo gravídico e, especialmente, diminuir o risco de baixo peso ao nascer do concepto.

SINISTERRA R., O.T. et al. [Maternal anemia and undernourishment and their relation to birth-weight]. Rev. Saúde públ., S. Paulo, 25: 193-7, 1991. With a view to evaluating the effect of anemia and maternal undernourishment on birth weight, a sample of 691 pregnant women, from an assistential maternity hospital, in S.Paulo, Brazil, were studied at delivery. Anemia was found in $29.1 \%$ and undernourishment in $17.2 \%$ of the women, though no association was detected between these nutritional deficiences. Neither was any association detected between institutional ante-natal care and anemia or undernourishment. Among single newborns the incidence of low birth weight (LBW) was of $12.9 \%$. LBW was observed in $23.6 \%$ of children born to women who came to the end of their pregnancy with a low weight for height ratios while among adequate weight for height women the percentage was 10.8 . Children of women who remained undernourished throughout their pregnancy had a relative risk of being born with LBW 2.8 times grater than the children of those who recovered normal weight by the end of their pregnancy. Our results emphasize the need for implementation of nutritional status control activities in programs of ante-natal care.

Keywords: Anemia, epidemiology. Nutritional status. Birth weight. Prenatal care

\section{Referências Bibliográficas}

1. ANTONOV, A. N. Children born during siege of Leningrad in 1942. J. Pediat., 30:250-9, 1947

2. BAKER, S.J. \& DEMAEYER, E. M. Nutritional anemia: its understanding and control with special reference to the work of the World Health Organization. Amer. J. clin. Nutr., 32:368-417, 1979.

3. BARROS, F.C. et al. Bajo peso al nacer en el Municipio de Pelotas, Brasil: factores de riesgo. Bol. Ofic. sanit. panamer., 102:541-54, 1987.

4. FLEMING, A.F. et al. Effects of iron and folic acid an - antenatal supplements on maternal haematology and fetal wellbeing. Med. J. Aust., 2:429-36, 1974.

5. HARRISON, K.A. \& IBEZIAKO, P.A. Maternal anaemia and fetal birthweight. J. Obstet. Gynaec. Brit. Cwlth., 80: 798-804, 1973.

6. HENNEKENS, C.H. \& BURING, I.E. Epidemiology in medicine. Boston, Little, Brown, 1987.

7. INTERNATIONAL NUTRITIONAL ANEMIA CONSULTATIVE GROUP. Iron deficiency in women. New York, Nutrition Foundation, 1981.

8. JACKSON, R.T . et al. Anemia of pregnancy in Leberia, West Africa: a therapeutic trial. Amer. J. clin. Nutr., 35:710-4, 1982.

9. MITCHELL, M.C. \& LERNER, E. Factors that influence the outcome of pregnancy in middle class women. $J$. Amer. diet. Ass., 87: 731-5, 1987.

10. ORGANIZAÇÃO MUNDIAL DA SAÚDE. Grupo Cientifico sobre Anemias Nutricionales, Ginebra, 1967. Informe. Ginebra, 1968. (Serie de Informes Técnicos, 405).

11. ORGANIZAÇÃO MUNDIAL DA SAÚDE. Reunião Mixta ADI/OIEA/OMS sobre Lucha contra la Anemia Nutricional, Especialmente contra la Carencia de Hierro, Ginebra, 1974. Informe. Ginebra, 1975. (Serie de Informes Técnicos, 580). 
12. PALGUi, A. et al. Anemia of pregnancy: evaluation of the effectiveness of routine dietary suplementation program in an Israeli community. Amer. J. publ. Hlth, 71: 736-9, 1981.

13. ROSSO, P. Nutrición e intercambio materno-fetal una perspectiva diferente. Arch. lat.-amer. Nutr., 27(supl. 1): $134-46,1977$.

14. ROYSTON, E. The prevalence of nutritional anaemia in developing coutries: a critical review of available information. Wld Hlth Statist. Quart., 35(2):52-75, 1982.

15. SECRETARIA DE ESTADO DA SAÚDE. Sub-Programa de Saúde da Mulher. Pré-natal normal. São Paulo, Grupo de Saúde da Mulher, s.d.

16. SEMINÁRIO sobre Anemias Nutricionais no Brasil, Brasília, 1977. Relatório final. Brasília, INAN, 1977. (Relatório Ténico, 21/77).
17. SIQUEIRA, A.A. F. et al. Influência da altura e ganho de peso materno e da idade gestacional sobre o peso do recém-nascido: estudo de três grupos de gestantes normais. Rev. Saúde públ., S. Paulo, 9:331-42, 1975.

18. SIQUEIRA, A.A.F. Indicadores de risco de peso inadequado ao nascer. São Paulo, 1981. [Tese de Livre Docência - Faculdade de Saúde Pública da USP].

19. SMITH, C.A. Effects of maternal undernutrition upon the newborn infant in Holland (1944 - 1945). $J$. Pediat., 30:229-43, 1947.

20. SZARFARC, S.C. Anemia ferropriva em parturientes e recém-nascidos de um grupo populacional de baixo nível sócio econômico de São Paulo. São Paulo, 1972. [Tese de Doutoramento - Faculdade de Saúde Pública da USP].

$$
\begin{array}{r}
\text { Recebido para publicação em } 7 / 12 / 1989 \\
\text { Reapresentado em 8/3/1991 } \\
\text { Aprovado para publicação em 14/3/1991 }
\end{array}
$$

